\title{
Características y disparidades entre sub-especialidades: un estudio de caso con grupos de Biotecnología
}

\author{
Fernanda Morillo*, Javier Aparicio*
}

Resumen: El objetivo de este trabajo es el estudio de las características y disparidades entre las sub-especialidades existentes dentro de categorías interdisciplinares, planteando la necesidad de identificar y evaluar por separado cada una de ellas. Se analizan los años 2003-2006, a través de las bases de datos del Web of Science (WoS), y se exploran las posibles diferencias entre grupos de investigación de Biotecnología de la Comunidad de Madrid según su perfil de especialización. Esta región española es la de mayor producción en Biotecnología (27\%) y dicha categoría presenta interacciones con otras disciplinas como Microbiología, Bioquímica y Alimentos. Por este motivo, el estudio de grupos incluye, en un segundo análisis, no sólo los documentos de esta categoría, sino también todas las publicaciones de los investigadores principales, independientemente de su disciplina. Con estos datos, se examina la influencia de la especialización en las características de los grupos, sus patrones de colaboración y su impacto. Se identifican tres tipologías según las sub-especialidades: $a$ ) grupos del NÚCLEO (publicando el 50\% o más de sus documentos en Biotecnología); $b$ ) grupos de tipo BÁSICO (publicando en Bioquímica y otras disciplinas básicas), y c) grupos de tipo MEDIO (publicando en Alimentos y disciplinas menos básicas). Por medio de pruebas estadísticas, se encontraron diferencias significativas entre estos conjuntos en relación con su producción e impacto. Se concluye que estas disparidades deberían ser tenidas en cuenta al evaluar la investigación que se produce en categorías interdisciplinares como la Biotecnología.

Palabras clave: Indicadores bibliométricos, sub-especialidades, evaluación, grupos de investigación, Biotecnología.

\section{Characteristics and disparities among sub-specialities: a case study of Biotechnology research teams}

Abstract: The objective of this work is the study of the characteristics and disparities among the existing sub-specialities within interdisciplinary categories, putting forward the need to identify and assess each one separately. The Web of Science (WoS) databases were examined for the years 2003-2006, in order to explore the possible differences between biotechnology research teams in Madrid according to their specialization profile. This

* Instituto de Estudios Documentales sobre Ciencia y Tecnología (IEDCYT) Centro de Ciencias Humanas y Sociales (CCHS-CSIC), Madrid, España. Correo-e: fernanda.morillo@cchs.csic.es, javier. aparicio@cchs.csic.es.

Recibido: 24-02-2011; 2. ${ }^{\text {a }}$ versión: 29-04-2011; 3. ${ }^{a}$ versión: 24-05-2011; 4. ${ }^{\text {a }}$ versión: 29-06-2011; aceptado: 30-06-2011. 
Spanish region has the highest output in biotechnology (27\%), a category that interacts with other disciplines, such as microbiology, biochemistry and food science. For this reason, the study of teams includes, in a second analysis, not just the documents in this category, but also publications of the team leaders, regardless of their discipline. With these data, the influence of the specialization on the teams' characteristics, collaboration patterns and impact is discussed. Three typologies are identified according to subspecialities: a) CORE teams (publishing 50\% or more in biotechnology); b) BASIC teams (publishing in biochemistry and other basic disciplines); and c) MEDIUM teams (publishing in food science and other less basic disciplines). By means of statistical tests, significant differences in output and impact were found among them. The article concludes that these disparities should be taken into account when assessing categories of interdisciplinary research such as biotechnology.

Keywords: bibliometric indicators, sub-specialities, assessment, research teams, biotechnology.

\section{Introducción y objetivos}

Una característica importante de la ciencia actual es el creciente papel de la colaboración y del trabajo en grupo. Entre los factores subyacentes que promueven la colaboración podemos mencionar los económicos (coste de los equipos), geográficos (proximidad), científicos (sinergias) y factores políticos (Beaver, 2001). La colaboración es fomentada por los gobiernos debido a que se presume que tiene efectos positivos en la creación de nuevos conocimientos y en el avance de la ciencia, y sobre todo para hacer frente a la creciente especialización y complejidad de la investigación científica. Según Katz y Martin (1997) los colaboradores normalmente serán aquellos que trabajan juntos a lo largo de un proyecto o durante una parte importante del mismo, los que aparecen en la propuesta original de investigación o los responsables de alguno de los elementos principales de la misma. En algunos casos también se puede incluir a los responsables de la hipótesis o a los gestores del proyecto (p. ej., investigadores principales o jefes de equipo). Desde el punto de vista bibliométrico, la colaboración se puede analizar a través de la múltiple autoría en las publicaciones científicas, tanto de investigadores individuales como de sus centros de pertenencia. El análisis mediante indicadores bibliométricos permite observar la estructura y dinámica de la colaboración a distintos niveles de agregación (macro, meso y micro), explorar los posibles beneficios de la colaboración y, en definitiva, aportar datos de interés para la política científica de instituciones y países.

La relevancia adquirida por los grupos es tal, que en muchas disciplinas han pasado a considerarse la mínima unidad del sistema investigador. Los grupos son hoy una pieza clave en el desarrollo de la investigación en gran parte de las disciplinas científicas y tecnológicas, mientras que el trabajo individual prevalece en algunas áreas de las ciencias sociales y, sobre todo, en humanidades. El interés de los estudios a nivel "micro" es diverso. Por un lado, el análisis de la actividad de los grupos a través de indicadores bibliométricos permite profundi- 
zar en el estudio de la estructura y dinámica del proceso investigador, analizado en lo que es su mínima unidad de funcionamiento y complementando otros enfoques, como los procedentes de la sociología de la ciencia. Pero además, estos estudios pueden aportar datos de interés para la política científica de un país, como, por ejemplo, la identificación de los grupos de excelencia en una determinada área científica. Estas razones explican un creciente interés por el estudio de los grupos de investigación desde distintas disciplinas y perspectivas (Bordons y otros, 1995 y 2005; Vinkler, 2000; Rey-Rocha y otros, 2006; van Raan, 2006).

El objetivo de este trabajo es estudiar las características de los grupos de investigación en función de sus sub-especialidades de publicación. Se ha seleccionado la Comunidad de Madrid debido a su papel predominante en la investigación científica en el conjunto de España (Morillo y De Filippo, 2009a). Por su parte, la Biotecnología siempre se ha considerado interdisciplinar de modo intuitivo, pero también a través de estudios concretos que analizan sus relaciones con otras categorías (Morillo y otros, 2003), lo que la hace candidata ideal como estudio de caso para la identificación de sus sub-especialidades. Para la evaluación de la investigación interdisciplinar (Laudel, 2006) resulta ventajoso mantener los criterios tradicionales para que las evaluaciones puedan compararse y la legitimidad de la investigación interdisciplinar no corra peligro por la creación de estándares especiales para su revisión. Klein (2008), por su parte, opina que no se deberían imponer metodologías ni impedir la evaluación a la espera de un método único y universal de medición que sería la antítesis de la multidimensionalidad de la propia investigación. Langfeldt (2006), en una postura intermedia, señala que es importante que la selección de revisores sea adecuada y se eviten conflictos, que se tenga claro a priori qué tipo de investigación se va a promover (convencional o interdisciplinar), y que se complemente la evaluación por pares con otros comités cuando sea preciso.

Dado que la Biotecnología es una categoría interdisciplinar, su delimitación no es sencilla y puede abordarse desde diversos ángulos o métodos. Han sido múltiples los autores que se han preocupado por el estudio de sus características y de su producción científica y tecnológica (por citar algunos: Rip y Courtial, 1984; McCain, 1995; Hinze y Grupp, 1996; Leydesdorff y Heimeriks, 2001; Dalpé, 2002; Casper y Murray, 2005; Frenken y otros, 2005; Albert y otros, 2007; Frenken y otros, 2010; Glänzel y Zhou, 2011). En el presente artículo se ha optado por el empleo de la clasificación en disciplinas de la base de datos WoS, multidisciplinar e internacional, que además permite la obtención de indicadores de visibilidad o impacto. Los antecedentes de este trabajo se encuentran, por una parte, en un estudio financiado por un proyecto de investigación realizado para la Comunidad de Madrid (Morillo y otros, 2009b) y, por otra, en resultados preliminares presentados en un congreso internacional (Morillo y otros, 2009c).

La identificación de grupos de investigación desarrollada en este trabajo se basa en el análisis de coautoría. Se considera que los autores que firman juntos un porcentaje importante de sus documentos son miembros de un equipo o grupo de investigación. Diferentes trabajos han abordado el estudio de grupos des- 
de una perspectiva bibliométrica, preocupándose por la localización de grupos de excelencia y por el estudio de su rendimiento y características dinámicas y estructurales, que son factores clave para tener éxito en la ciencia (Bordons y otros, 1995; Calero y otros, 2006; Seglen \& Aksnes, 2000; Börner y otros, 2005).

Previa normalización de nombres de autores y de sus lugares de trabajo, para caracterizar los grupos, se obtienen indicadores de producción científica, impacto esperado (factor de impacto) y observado (citas recibidas) y, colaboración (nacional e internacional). Además, a través del análisis de redes se estudian las relaciones entre autores (densidad, grado, distancia, etc). Con todos estos datos se exploran las diferencias existentes entre tipologías según las sub-especialidades, ya que la elaboración de indicadores pertinentes para la evaluación de grupos de investigación es esencial. La hipótesis que se plantea en este trabajo es que coexisten diversas sub-especialidades, dentro de categorías inter o multidisciplinares como la Biotecnología, que pueden detectarse por medios bibliométricos y que tienen características drásticamente distintas que afectan a la evaluación de los grupos de investigación. En este sentido, se pretende analizar la estructura y dinámica de los grupos identificando posibles diferencias según su perfil de especialización. Para este objetivo se analiza un estudio de caso con los grupos de investigación de Biotecnología de la Comunidad de Madrid en el período 2003-2006.

\section{Materiales y métodos}

En este estudio se utilizan las bases de datos bibliográficas internacionales de Thomson Reuters (antes ISI): Science Citation Index (SCI), Social Sciences Citation Index (SSCI) y Arts \& Humanities Citation Index (A\&HCI), en la versión Web of Science (WoS). Estas bases de datos, procedentes de EE.UU., son multidisciplinares y recogen más de 9.000 revistas de la corriente principal de la ciencia internacional clasificadas en una o varias categorías temáticas. Para la obtención de grupos de investigación, se analiza la producción científica de la región española de Madrid, en las revistas incluidas por Thomson Reuters en la categoría denominada Biotecnología, durante 2003-2006. Este trabajo no pretende ser exhaustivo. Al seleccionar como punto de partida la categoría "Biotecnología" sólo se pretende localizar una parte importante de la producción de los grupos de biotecnólogos de Madrid, pero no la de aquéllos que publican en revistas de cualquiera de las diversas disciplinas relacionadas con la Biotecnología.

La metodología utilizada para el análisis de grupos se ha desarrollado por el grupo ACUTE del IEDCYT y se basa en programas que interactúan sobre la descarga de publicaciones científicas para: $a$ ) obtener la frecuencia de aparición de cada autor ligado a su lugar de trabajo; $b$ ) analizar la frecuencia de aparición de parejas de autores; $c$ ) agrupar autores en función de su frecuencia de coautoría. Una aplicación informática preliminar a la aquí utilizada se describió en un estudio previo (Bordons y otros, 1995). En el desarrollo de esta metodología es 
muy importante identificar correctamente a cada autor para calcular de forma precisa su productividad y, por ello, una fase esencial del proceso es la normalización de nombres de autores y de centros.

Una vez realizada la normalización de datos, se procede al cálculo de frecuencias de coautoría y al establecimiento de agrupaciones de autores. Cada grupo se identifica por un investigador principal (IP) o líder, que es el autor más productivo dentro de un grupo, al menos desde el punto de vista bibliométrico. Para perfilar los grupos se han probado distintas condiciones de agrupación de autores seleccionándose, finalmente, las que han producido mejores resultados: a) nivel de coautoría: un autor debe firmar al menos el 66\% de su producción con un IP, para ser asignado a su grupo; b) tamaño de grupo: un grupo puede estar formado por 2 o más autores; c) producción mínima de un autor para figurar como IP: se considera que debe de tener al menos 4 documentos en todo el período.

Al analizar los resultados se comprueba que, aunque los grupos formados son válidos, la producción de los investigadores principales en revistas incluidas por Thomson Reuters, dentro de la disciplina Biotecnología, respecto a su producción total, queda, en muchos casos, por debajo del 50\%. Esto se debe a que los investigadores publican gran parte de sus documentos en revistas clasificadas en otras categorías, que se quedan fuera de este análisis, aunque en muchos casos son de interés para el área. Por este motivo, al anterior conjunto de documentos, se añade la totalidad de la producción, en todas las disciplinas, de los investigadores principales, dado que, con los umbrales establecidos en este trabajo, se supone que toda la producción del grupo quedará así recogida. Este paso aumenta de forma significativa el número de autores y documentos, realizándose por ello un segundo análisis de grupos.

Los grupos se identifican por el nombre del investigador principal (IP) y por su centro de trabajo y se describen a través de una serie de indicadores: tamaño del grupo (número de miembros, teniendo en cuenta la frecuencia de coautoría); producción (número de documentos publicados por el grupo); productividad (producción/tamaño); promedio de citas/documento; FI medio de 2005; porcentaje de documentos en colaboración nacional; porcentaje de documentos en colaboración internacional; porcentaje de documentos en revistas de la disciplina Biotecnología, etc. Aunque no se utiliza como variable de selección para las tipologías según sub-disciplinas, se calcula el nivel medio de investigación para cada una, estableciendo similitudes con la definición de las mismas. El nivel de investigación es un índice ofrecido por The Patent Board que varía de 1 (investigación aplicada) a 4 (investigación básica) (Noma, 1986).

Para examinar los resultados, además de los indicadores cuantitativos y semi-cualitativos mencionados, se han utilizado técnicas de análisis de redes sociales, empleando representaciones gráficas y medidas descriptivas de redes, mediante el programa Pajek (Batagelj y Mrvar, 1998). Este software permite representar las redes de colaboración y coautoría utilizando diferentes análisis y medidas, entre los que destacan: 
- Densidad. Es una medida referente al total de la red. Representa el número de vínculos establecidos entre los nodos en relación al número máximo que pudiera establecerse y está asociada al tamaño de la red. Se utiliza para medir la cohesión de la red.

- Grado o grado de centralidad. Identifica el número de nodos (autores) con los que un nodo (autor) está colaborando.

- Grado Medio de la red. Es la media de los grados de cada grupo o autor y describe a la totalidad de la red. En el estudio, se utiliza para comparar la cohesión o conectividad de distintas redes, por ser más precisa que la densidad, ya que no depende del tamaño de la red (De Nooy y otros, 2005).

- Distancia media entre nodos. Mide la distancia media del camino más corto entre pares de nodos de la red.

Se analizan la estructura y el rendimiento de los grupos (actividad, impacto, colaboración) de acuerdo con su perfil temático de investigación. Los principales aspectos estudiados incluyen:

1. Estructura del grupo: tamaño del grupo y densidad o cohesión.

2. Rendimiento del grupo: producción científica, productividad, indicadores de impacto (número de citas, promedio de citas/documento, porcentaje de documentos en revistas de alto factor de impacto), y patrón de colaboración (autores e instituciones por documento, tasas de colaboración nacional e internacional).

3. Especialización del grupo: se explora la utilidad de los diferentes indicadores para caracterizar el perfil de especialización de los grupos (disciplina principal de publicación, porcentaje de los documentos en Biotecnología, e índice de interdisciplinariedad - la dispersión de las publicaciones en las disciplinas se mide a través del índice de Pratt-).

Por último, gracias al software SPSS 19.0, se utilizan las pruebas estadísticas no paramétricas de Kruskal-Wallis para comprobar si las tipologías son significativamente distintas para diversas variables y se aplica el análisis de componentes principales para simplificar la información procedente de las mismas. De todas las posibles combinaciones se seleccionan las que explican una proporción aceptable de la varianza global, permitiendo que pocas variables sean útiles al estudio y favorezcan la interpretación de los resultados obtenidos (IBM SPSS Statistics Base 19, 2010; Pérez, 2005).

\section{Resultados}

En el período 2003-2006 en las bases WoS, Madrid es la Comunidad Autónoma española con mayor producción en Biotecnología (711 documentos, de los cuales 654 son artículos, que representan un 27\% de la producción española en 
el área). Las interacciones de la Biotecnología con otras disciplinas se ponen de manifiesto a través del estudio de la multi-asignación de sus revistas en otras disciplinas (clasificación WoS). Se detectan así las relaciones de la Biotecnología con disciplinas de tipo básico (Microbiología o Bioquímica) o aplicado (Ciencia y Tecnología de Alimentos), observando que todas estas interacciones son de gran impacto, ya que hay un elevado porcentaje de artículos publicados en revistas del primer cuartil, es decir, con FI alto (tabla I). En esta tabla se presentan sólo los 654 artículos, por considerar que únicamente éstos son susceptibles de citación y sólo se muestran las disciplinas de mayor producción.

\section{TABLA I}

Principales disciplinas relacionadas con la Biotecnología en la Comunidad de Madrid por la multi-asignación de revistas (>65 artículos)

\begin{tabular}{l|c|c|c|c|c|c|c}
\hline \multicolumn{1}{c|}{ Temas } & $\mathbf{2 0 0 3}$ & $\mathbf{2 0 0 4}$ & $\mathbf{2 0 0 5}$ & $\mathbf{2 0 0 6}$ & Arts. & $\mathbf{\%}$ & $\begin{array}{c}\text { \% Art. } \\
\text { Q1 }\end{array}$ \\
\hline Microbiología & 22 & 32 & 36 & 25 & 115 & 17,6 & 53,0 \\
\hline Ciencia y Tecnología de Alimentos & 18 & 36 & 22 & 21 & 97 & 14,8 & 77,6 \\
\hline Biométodos & 11 & 22 & 23 & 22 & 78 & 11,9 & 47,3 \\
\hline Bioquímica y Biología Molecular & 23 & 17 & 17 & 17 & 74 & 11,3 & 51,9 \\
\hline Genética y Herencia & 19 & 18 & 16 & 16 & 69 & 10,6 & 64,6 \\
\hline Virología & 11 & 22 & 17 & 16 & 66 & 10,1 & 76,8 \\
\hline
\end{tabular}

\% calculado sobre los 654 artículos de Biotecnología. \% Art. Q1: \% de artículos en revistas del primer cuartil o de alto factor de impacto.

El análisis de grupos se realiza, en un primer momento, sobre las publicaciones de la Comunidad de Madrid en las revistas de Biotecnología (Análisis I) pero, al observar que los principales investigadores del área publican una parte importante de sus documentos en revistas de otras categorías, se realiza un segundo estudio. Este estudio parte de las revistas de Biotecnología y añade todas las publicaciones de los líderes de los grupos, independientemente de su revista de publicación (Análisis II), lo que aporta una mayor cobertura de la actividad grupal.

En el estudio de Morillo y otros (2009b), el Análisis I, centrado en las publicaciones en revistas de Biotecnología, permite identificar 45 grupos y, a través del Análisis II, que incluye todas las publicaciones de los IP independientemente de su disciplina, se detectan 55 grupos, no teniendo en cuenta los IP extranjeros. El mayor incremento en el número y porcentaje de grupos (casi un 4\%) se produce en el sector CSIC (tabla II). Además, la cohesión y densidad de la red de colaboración entre grupos aumentan en este segundo análisis. Incluyendo todas las publicaciones de los IP, con independencia de su campo de publicación, la producción de los grupos de Madrid crece a 1398 documentos (el doble del 
Análisis I). En este segundo análisis, el tamaño de los grupos varía de 2 a 43 miembros y su producción varía de 4 a 103 documentos por grupo. Por otro lado, al estudiar la situación de los grupos en la red regional se observa que hay algunos grupos aislados, mientras que otros con alta centralidad desempeñan un papel importante conectando grupos.

TABLA II

Análisis I vs. Análisis II. Grupos por sector institucional

\begin{tabular}{l|c|c|c|c}
\hline \multirow{2}{*}{ Sector institucional } & \multicolumn{2}{c|}{ Análisis I } & \multicolumn{2}{c}{ Análisis II } \\
\cline { 2 - 5 } & $\boldsymbol{N}$ grupos & \% grupos & $\boldsymbol{N}$ grupos & \% grupos \\
\hline CSIC & 22 & 48,89 & 29 & 52,73 \\
\hline Universidad & 11 & 24,44 & 12 & 21,82 \\
\hline Otros OPI & 9 & 20,00 & 9 & 16,36 \\
\hline Empresas & 1 & 2,22 & 2 & 3,64 \\
\hline Sector Sanitario & 1 & 2,22 & 2 & 3,64 \\
\hline Administración & 1 & 2,22 & 1 & 1,82 \\
\hline Total & 45 & & 55 & \\
\hline
\end{tabular}

Antes de acometer el estudio del perfil temático (de este artículo) y, para seleccionar los indicadores más pertinentes, se toma como referencia los tres grupos de Biotecnología, examinados con detalle en Morillo y otros (2009b) (Análisis II). Se eligieron por su elevado número de documentos y su alta especialización en Biotecnología (bien porque tienen un elevado porcentaje de producción en dicha disciplina WoS o bien porque su centro de pertenencia está claramente especializado en el tema). De estos grupos, sólo se exponen las mayores diferencias de comportamiento, justificadas en parte por su distinta subespecialidad, cuyo estudio es el objetivo final del presente artículo. Uno de los grupos analizados tiene un IP del Instituto de Catálisis y Petroleoquímica (llamado aquí IP1) y, los otros dos, tienen IP del Centro Nacional de Biotecnología (identificados en este estudio como IP2 e IP3). Se observan diferencias importantes en la estructura de los grupos. El grupo del IP1 cuenta con una estructura bicéfala (dos autores muy productivos con un peso similar de producción y similar liderazgo), mientras que los grupos del IP2 y del IP3 presentan un núcleo muy fuerte y central dentro de sus grupos, teniendo el IP3 especial relevancia como intermediario del resto de los autores del grupo (figura 1).

Las colaboraciones entre grupos y la colaboración con autores extranjeros se analizan tanto en términos de intensidad como de variedad de colaboradores. El grupo del IP2 muestra la mayor colaboración con otros grupos, en cuanto a variedad de relaciones, mientras que el grupo del IP1 se caracteriza por una alta intensidad de relaciones con alguno de sus grupos colaboradores. Además, se 


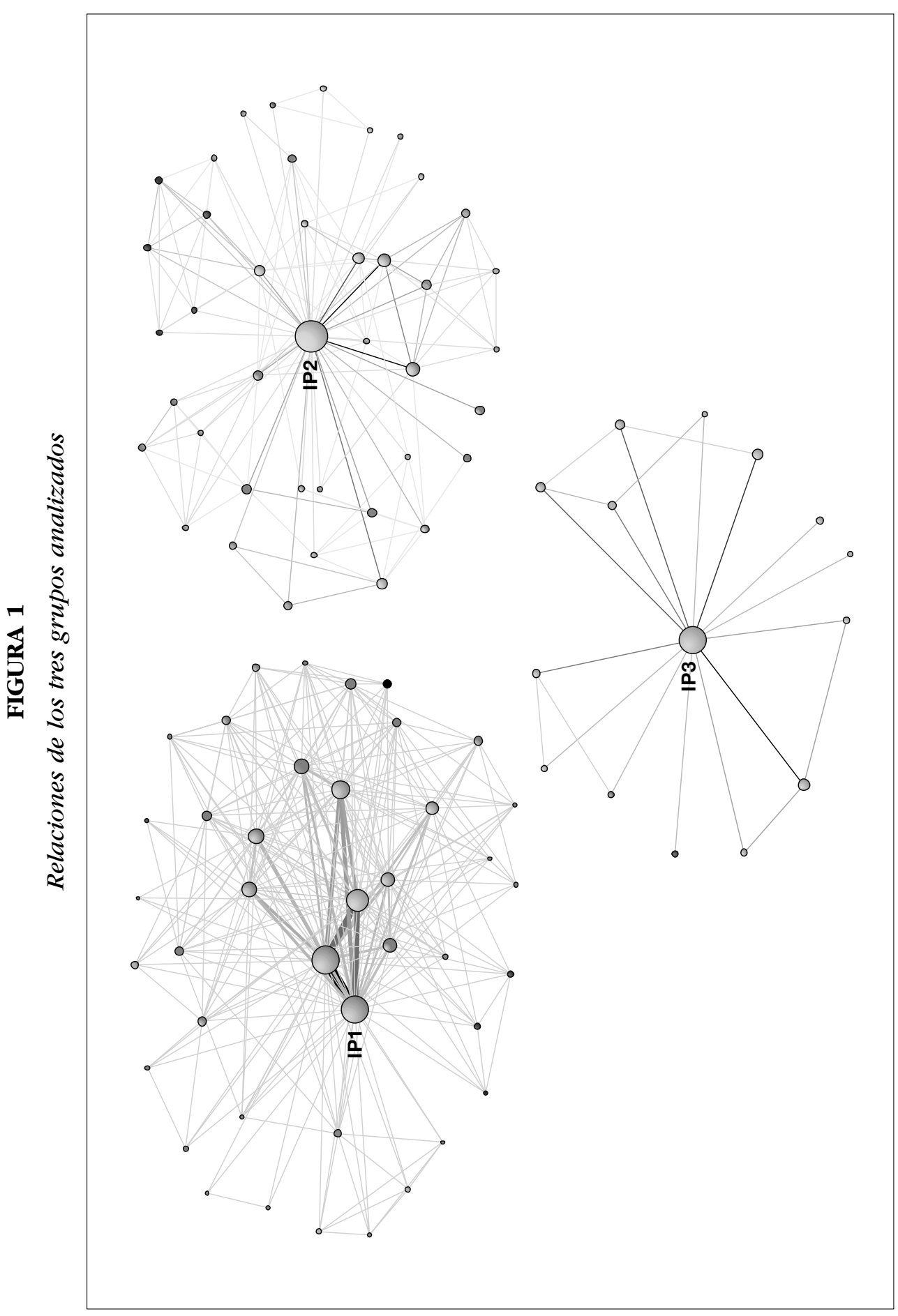

Rev. Esp. Doc. Cient., 34, 4, octubre-diciembre, 563-580, 2011. ISSN: 0210-0614. doi: 10.3989/redc.2011.4.837 
estudia el perfil temático de actividad de los grupos y las características de su producción. El grupo del IP1 muestra una alta especialización en la disciplina de Biotecnología de WoS (64\%) y, sólo en segundo lugar, en Bioquímica (24\%), mientras que el grupo del IP2 muestra una mayor especialización en Bioquímica (52\%) y menor en Biotecnología (27\%). El grupo del IP3, por su parte, destaca en Microbiología (Figura 2). De hecho, se observa mayor impacto del grupo del IP2 frente al del IP1 ( 6 citas por documento frente a 4 citas por documento, respectivamente) influyendo posiblemente el carácter más básico de la investigación en Bioquímica o el tamaño del área. Sin embargo, dado que los tres grupos tienen resultados positivos, la comparación entre indicadores, sin considerar la especialización temática, podría llevar a conclusiones erróneas.

FIGURA 2

Tres principales disciplinas WoS de los gupos IP1, IP2 e IP3

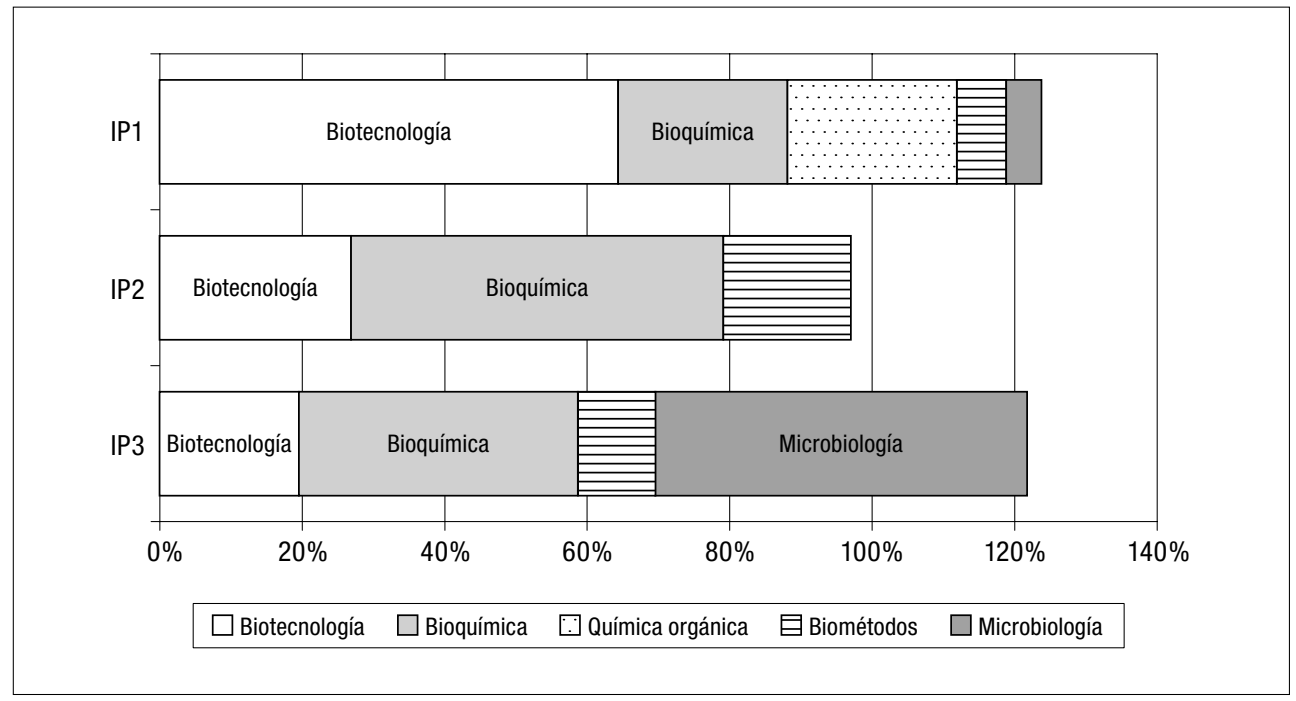

Por este motivo, se desea conocer hasta qué punto influye la sub-especialidad en las características del grupo, en sus patrones de colaboración y en su impacto. Para el estudio del presente artículo se parte de los 55 grupos de investigación estudiados en el Análisis II (2003-2006), eliminando uno de ellos, por carecer de publicaciones en la categoría de Biotecnología. En total quedan 54 grupos. Se realizan diversas pruebas de delimitación considerando la primera especialidad temática de cada grupo y, finalmente, se identifican tres tipologías de grupos: a) 19 grupos forman el NÚCLEO (los que publican el 50\% o más de sus documentos en Biotecnología); b) 19 grupos realizan investigación de tipo BÁSICO (los que publican principalmente en Bioquímica y otras disciplinas básicas), y c) 16 grupos 
se encuentran en el MEDIO (los que publican principalmente en Alimentos y disciplinas menos básicas). Por otra parte, como ya se comentó en la Metodología, se calcula y muestra el nivel medio de investigación para cada tipología como una característica más de las mismas.

Se parte de la hipótesis planteada de que estos tres conjuntos o tipologías se comportan de forma diferente debido a su distinta especialización y que, por tanto, los grupos deben ser analizados y evaluados de forma separada. Para contrastar esta hipótesis se analizan distintas variables para cada uno de los grupos. Se ejecuta la prueba estadística no paramétrica de Kruskal-Wallis para muestras independientes, para ver si los datos provienen o no del mismo conjunto con estas variables (tabla III):

1. Producción (total de documentos del grupo). Variable significativamente diferente en cada uno de los conjuntos analizados.

2. Tamaño (número de investigadores del grupo). Variable significativamente diferente en cada uno de los conjuntos analizados.

3. Productividad (relación entre la producción y el tamaño). No significativa.

\section{TABLA III}

\section{Prueba de Kruskal-Wallis}

\begin{tabular}{l|c|c|c}
\hline & Estadisticos de contraste & \\
\hline & Chi-cuadrado & gl & Sig. asintót. \\
\hline Producción & 12,355 & 2 & 0,002 \\
\hline Tamaño & 6,499 & 2 & 0,039 \\
\hline Productividad & 1,388 & 2 & 0,500 \\
\hline Densidad & 9,476 & 2 & 0,009 \\
\hline Índice de Pratt & 2,151 & 2 & 0,341 \\
\hline FI_Biotec. & 14,463 & 2 & 0,001 \\
\hline PN & 12,585 & 2 & 0,002 \\
\hline$\%$ Q1 & 11,937 & 2 & 0,003 \\
\hline Citas medias & 17,199 & 2 & 0,000 \\
\hline Citas medias Biotec. & 9,533 & 2 & 0,009 \\
\hline Media autores & 5,130 & 2 & 0,077 \\
\hline Media organismos & 4,453 & 2 & 0,108 \\
\hline$\%$ colaboración internacional & 9,296 & 2 & 0,010 \\
\hline \% colaboración nacional & 0,826 & 0,662 \\
\hline
\end{tabular}

a Variable de agrupación: tipos MEDIO, BÁSICO y NÚCLEO basados en sub-disciplinas.

PN: posición normalizada media del FI para comparar entre especialidades.

$\%$ Q1: \% de documentos en revistas del primer cuartil o de alto factor de impacto. 
4. Densidad (medida a través del número de relaciones de la red de autores de un grupo). Variable significativamente diferente en cada uno de los conjuntos analizados.

5. Índice de Pratt (concentración o dispersión de la producción en distintas disciplinas). No significativa.

6. Promedio de Factor de Impacto de la disciplina de Biotecnología. Variable significativamente diferente en cada uno de los conjuntos analizados.

7. Promedio de Posición Normalizada (PN) de las disciplinas en las que publican los grupos de investigación. Variable significativamente diferente en cada uno de los conjuntos analizados.

8. Porcentaje de documentos en el primer cuartil (\% Q1). Variable significativamente diferente en cada uno de los conjuntos analizados.

9. Promedio de citas de dicha producción. Variable significativamente diferente en cada uno de los conjuntos analizados.

10. Promedio de citas de la disciplina de Biotecnología. Variable significativamente diferente en cada uno de los conjuntos analizados.

11. Promedio de autores/organismos de dicha producción. No significativa.

12. Porcentaje de colaboración internacional. Variable significativamente diferente en cada uno de los conjuntos analizados.

13. Porcentaje de colaboración nacional. No significativa.

En resumen, se encontraron diferencias significativas entre tipologías con respecto a la estructura del grupo (densidad y tamaño del grupo) y algunas de las variables utilizadas para el rendimiento del grupo (número de documentos, indicadores de impacto y tasas de colaboración internacional). No se encontraron diferencias significativas, en cambio, respecto al índice de productividad, el promedio del número de autores y organismos y el tipo de colaboración nacional. El índice de Pratt tampoco demostró ser una buena medida para definir las tipologías.

Posteriormente, se agrupan todas las variables mediante un análisis de componentes principales (Varimax con normalización de Kaiser) en cuatro factores: tamaño/producción (con valores positivos para el índice de Pratt, el total de documentos y el tamaño del grupo, y valores negativos para la densidad); impacto esperado (Factor de impacto en la Biotecnología, posición normalizada de esta variable para comparar entre especialidades y porcentaje de documentos en revistas del primer cuartil o de alto factor de impacto); impacto observado (promedio de citas en Biotecnología y promedio de citas en todas las categorías); y colaboración (promedio del número de autores, de organismos y de tasas de colaboración nacional e internacional) (tabla IV). Estos cuatro factores explican casi el 77\% de la varianza, por lo que ofrecen una información muy fiable para la interpretación de los resultados.

Por medio de la prueba estadística no paramétrica de Kruskal-Wallis para muestras independientes, se encontraron diferencias significativas entre tipologías 


\section{TABLA IV}

Análisis factorial. Matriz de componentes rotados

\begin{tabular}{|c|c|c|c|c|}
\hline & \multicolumn{4}{|c|}{ Componente } \\
\hline & $\begin{array}{c}\text { Tamaño/ } \\
\text { producción }\end{array}$ & $\begin{array}{l}\text { Impacto } \\
\text { esperado }\end{array}$ & Colaboración & $\begin{array}{c}\text { Impacto } \\
\text { observado }\end{array}$ \\
\hline Índice de Pratt & 0,777 & & & \\
\hline Citas medias & & 0,550 & & 0,691 \\
\hline $\mathrm{PN}$ & & 0,869 & & \\
\hline$\%$ Q1 & & 0,867 & & \\
\hline FI_Biotec. & & 0,800 & & \\
\hline Citas medias Biotec. & & & & 0,825 \\
\hline Producción & 0,897 & & & \\
\hline Tamaño & 0,906 & & & \\
\hline Media autores & & & 0,533 & 0,401 \\
\hline Media organismos & & & 0,899 & \\
\hline Densidad & $-0,900$ & & & \\
\hline \% colaboración nacional & & & 0,638 & \\
\hline \% colaboración internacional & & & 0,658 & \\
\hline
\end{tabular}

Método de rotación: Normalización Varimax con Kaiser. La rotación ha convergido en 7 iteraciones. No se muestran valores inferiores a 0,4. PN: posición normalizada media del FI para comparar entre especialidades. $\%$ Q1: \% de documentos en revistas del primer cuartil o de alto factor de impacto.

TABLA V

Análisis II. Variables significativas por tipología de grupos

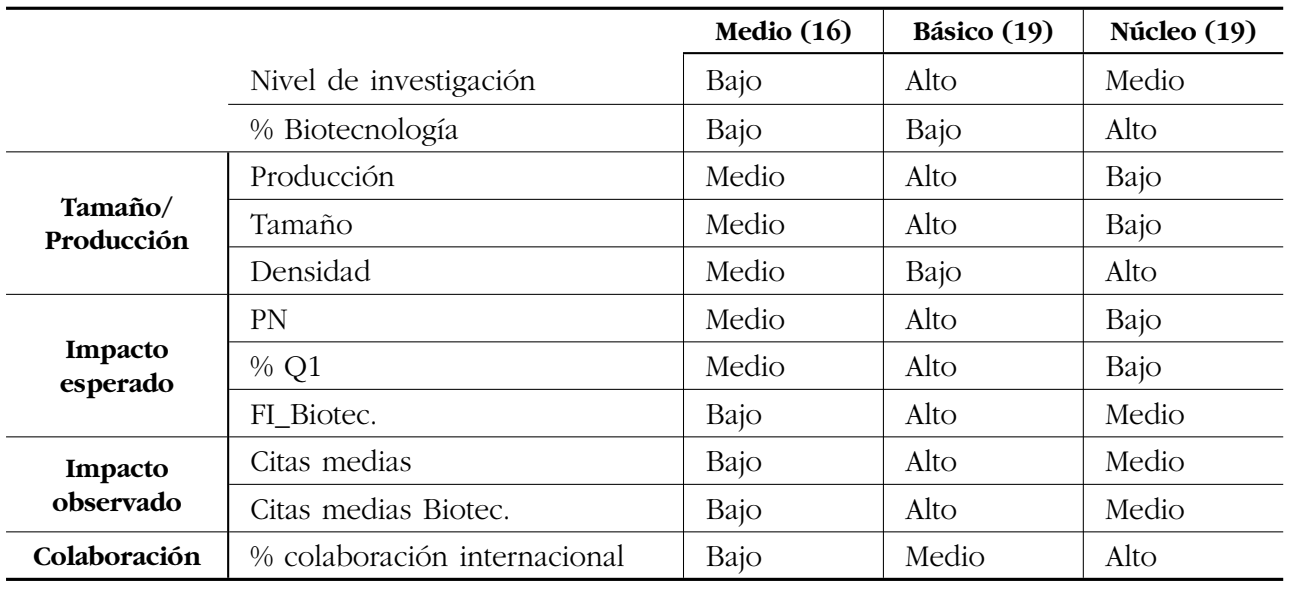

Nota: Tipo MEDIO (grupos que publican en Alimentos y disciplinas menos básicas), tipo BÁSICO (grupos que publican en Bioquímica y otras disciplinas básicas) y grupos del NúCLEO (con el 50\% o más de sus documentos en Biotecnología). PN: posición normalizada media del FI para comparar entre especialidades. $\%$ Q1: \% de documentos en revistas del primer cuartil o de alto factor de impacto. 
en relación con: el tamaño/producción y los impactos esperado y observado (tabla V). Utilizando la misma prueba comparando conjuntos dos a dos: los conjuntos MEDIO y BÁSICO vuelven a diferenciarse en las variables de impacto. Los conjuntos MEDIO y NÚCLEO se diferencian en tamaño/producción y también en impacto. Por último, los conjuntos BÁSICO y NÚCLEO se diferencian tanto en tamaño como en impacto (pero sólo el esperado).

Los grupos del tipo BÁSICO son activos en Bioquímica, Microbiología, Genética y Virología (todos ellos con un nivel de investigación promedio de 4). Los grupos del tipo MEDIO son activos en Alimentos, temas Medioambientales y Ciencias técnicas (todos ellos con un nivel de investigación promedio de 2,5). Los del tipo BÁSICO destacan en: tamaño/producción y en los impactos esperado y observado. Los grupos del NÚCLEO (con un nivel de investigación similar al tipo BÁSICO) muestran las puntuaciones más altas en colaboración pero las más bajas en tamaño/producción. Los del tipo MEDIO también tienen niveles medios de impacto esperado y de tamaño/producción y las puntuaciones más pequeñas en colaboración (tabla $\mathrm{V}$ y figura 3).

\section{FIGURA 3}

\section{Análisis de componentes principales: valores de las agrupaciones de variables para cada tipología}

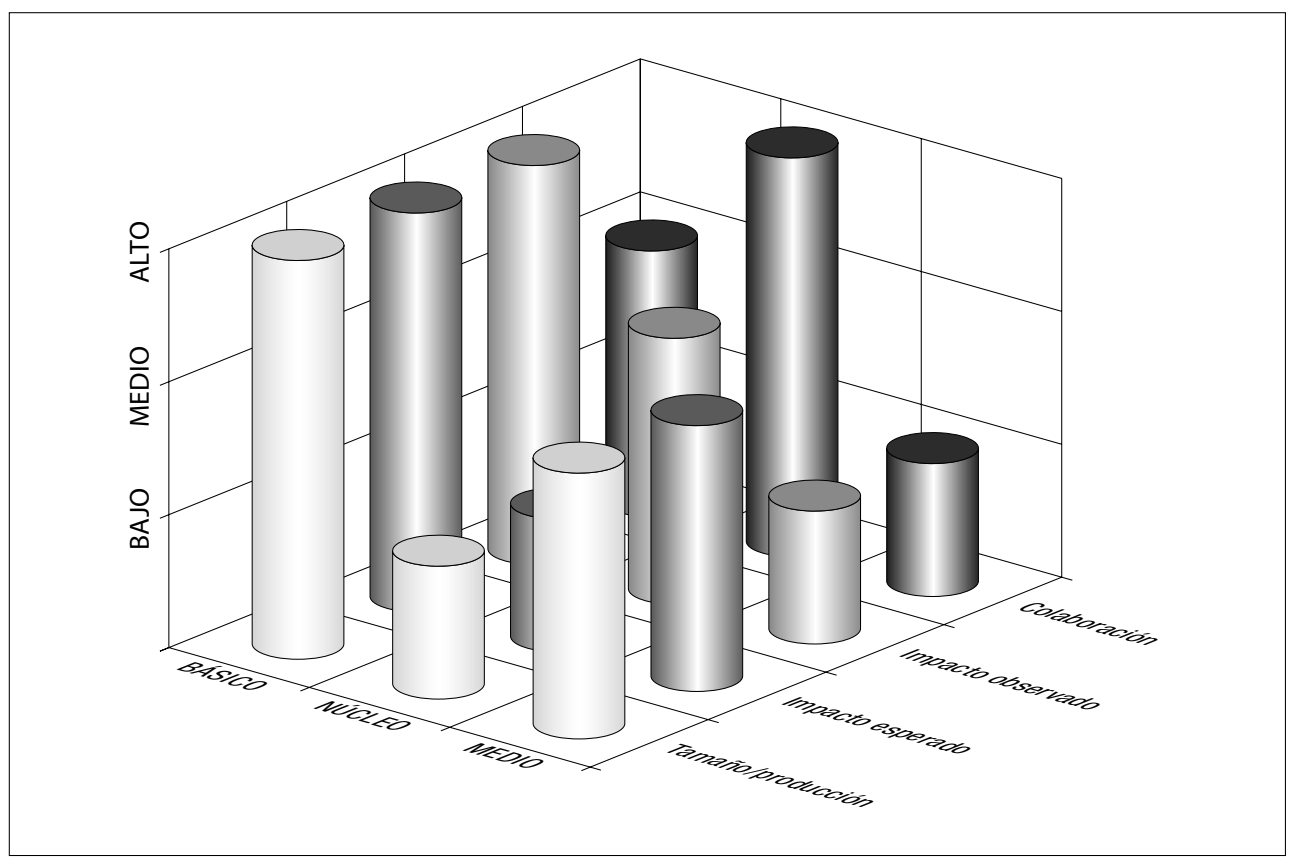

Nota: Tipo MEDIO (grupo que publican en Alimentos y disciplinas menos básicas), tipo BÁSICO (grupos que pulbican en Bioquímica y otras disciplinas básicas) y grupos del NÚCLEO (con el 50\% o más de sus documentos en Biotecnología). 


\section{Conclusiones y discusión}

El estudio de caso llevado a cabo ha puesto de manifiesto la coexistencia de sub-especialidades dentro de la disciplina de la Biotecnología, lo que es lógico dado el carácter multidisciplinar de esta categoría. Las metodologías utilizadas se han mostrado válidas para la clasificación de grupos de investigación por tipologías, según su sub-especialidad principal dentro de esta disciplina. La obtención de las categorías principales que sirven de diferenciación entre tipologías es rápida y sencilla. La importancia de este tipo de estudio viene dada por la necesidad de encontrar un método adecuado que pueda servir para evaluar la actividad que se produce en el seno de las categorías interdisciplinares de la forma más objetiva posible. Es necesario aplicar un método cuantificable que pueda servir para comparar elementos iguales y dé una idea precisa de qué se está midiendo y qué se está valorando. Esta preocupación se ha podido observar en otros trabajos, como el de Porter y otros (2010), en el que se muestra un estudio exploratorio para la identificación de perfiles de investigación, utilizando un software de minería de texto (text-mining), con el objeto de ofrecer una información más detallada a los evaluadores. Gracias a estos datos, los gestores podrán formar más fácilmente paneles de revisión e identificar investigaciones de carácter interdisciplinar.

La producción y las citas e impacto difieren de una sub-especialidad a otra dentro de la categoría WoS de Biotecnología, por lo que se confirma la hipótesis de la existencia de tipologías temáticas con características drásticamente distintas y, no sólo eso, sino que se observa que dichas tipologías pueden asimilarse en su comportamiento a otras categorías distintas de la Biotecnología. Por esa razón, se aconseja la identificación y evaluación independiente de la investigación que se lleva a cabo dentro de cada sub-especialidad. Dicha valoración podría agrupar tipologías iguales según la especialización principal de cada conjunto, evaluando la investigación en su categoría correspondiente, lo que permitiría aplicar métodos tradicionales en la valoración de categorías interdisciplinares. Múltiples han sido los estudios deseosos de encontrar un sistema adecuado de clasificación que permita las comparaciones del modo más apropiado posible y, dado que la actividad de los investigadores no suele limitarse a una sola disciplina, la determinación de su área de actividad es un tema trascendental en cuestiones de evaluación y de comparación de resultados (Glänzel y Schubert, 2003).

La revisión por pares es una práctica de evaluación de la investigación en la que un trabajo es evaluado por colegas que trabajan en temas similares. Sin embargo, hay una falta de criterio para priorizar la financiación de la investigación interdisciplinar. Por un lado, por la difícil identificación de lo que se está evaluando y, por otro, porque es complicado sintetizar diversas opiniones y reconciliar perspectivas. Los pocos estudios que analizan la investigación interdisciplinar tienden a favorecer la introducción de criterios especiales de evaluación, dando importancia a la bondad de la integración o al ajuste de los conocimientos procedentes de distintas áreas. Autores como Laudel (2006) consideran que 
es posible mantener criterios tradicionales de evaluación frente a autores como Klein (2008), que creen que un método único puede distorsionar la multidimensionalidad de la propia investigación. Langfeldt (2006), por su parte, señala la importancia de la evaluación tradicional siempre que pueda ser complementada cuando y dónde sea necesario.

Este artículo plantea una solución intermedia y menos costosa que las apuntadas en otros estudios. Aunque sólo se analice un colectivo pequeño, la Biotecnología de Madrid, se puede concluir que este método permite la identificación de tipologías homogéneas, para "Subdividir" el trabajo de investigación que se está examinando. De este modo, según cuál sea el tema principal, será posible redireccionar la evaluación a las comisiones especializadas pertinentes favoreciendo la adecuación y objetividad de la revisión. En el futuro se realizarán nuevos análisis de otras categorías similares (de carácter híbrido o interdisciplinar) para probar la disparidad de características entre sub-especialidades aplicando metodologías bibliométricas para la detección de tipologías dentro de cada categoría. La identificación de tipologías de sub-especialidades ayudará a la organización de paneles de evaluación de proyectos, centros o investigadores al permitir discernir cuál es el núcleo de actividad de cada una de ellas y valorar la investigación que se lleva a cabo más adecuadamente.

\section{Agradecimientos}

Queremos agradecer a nuestros colegas del grupo ACUTE (Análisis Cuantitativo en Ciencia y Tecnología), especialmente a Isabel Gómez, sus comentarios y su colaboración en el análisis de los datos.

\section{Bibliografía}

Albert, A.; Granadino, B., y Plaza, L. M. (2007). Scientific and technological performance evaluation of the Spanish Council for Scientific Research (CSIC) in the field of Biotechnology. Scientometrics, 70 (1): 41-51.

Batagelj, V., y Mrvar, A. (1998). Pajek: a program for large network analysis. Connections (Tor.), 21 (2), 47-57.

Beaver, D. De B. (2001). Reflections on scientific collaboration (and its study): past, present and future. Scientometrics, 52 (3), 365-377.

Bordons, M.; Morillo, F.; Fernández, M. T.; Gómez, I.; León, M., y Martín D. (2005). La investigación matemática española de difusión internacional. Estudio bibliométrico 1996-2001. CSIC.

Bordons, M.; Zulueta, M. A.; Cabrero, A., y Barrigón, S. (1995). Identifying research teams with bibliometric tools. En: Koenig, M. E. D. y Bookstein, A. (eds.), Fifth International Conference of the International Society for Scientometrics and Informetrics. Learned Information, Medford.

578 Rev. Esp. Doc. Cient., 34, 4, octubre-diciembre, 563-580, 2011. ISSN: 0210-0614. doi: 10.3989/redc.2011.4.837 
Börner, K.; Dall'Asta, L.; Ke, W., y Vespignani, A. (2005). Studying the Emerging Global Brain: Analyzing and Visualizing the Impact of Co-Authorship Teams. Complexity, 10 (4), 57-67.

Calero, C.; Buter, R.; Cabello Valdés, C., y Noyons, E. (2006). How to identify research groups using publication analysis: an example in the field of nanotechnology. Scientometrics, 66 (2), 365-376.

Casper, S., y Murray, F. (2005). Careers and clusters: analyzing the career network dynamic of biotechnology clusters. Journal of Engineering and Technology Management, 22, 51-74.

Dalpé, R. (2002). Bibliometric analysis of biotechnology. Scientometrics, 55 (2): 189-213.

De Nooy, W.; Mrvar, A., y Batagelj, V. (2005). Exploratory Network Analysis with Pajek. Cambridge University Press.

Frenken, K.; Hölzl, W., y de Vor, F. (2005). The citation impact of research collaborations: the case of European biotechnology and applied microbiology (1988-2002). Journal of Engineering and Technology Management, 22, 9-30.

Frenken, K.; Ponds, R., y Van Oort, F. (2010). The citation impact of research collaboration in science-based industries: A spatial-institutional analysis. Papers in Regional Science, 89 (2): 351-371.

Glänzel, W., y Schubert, A. (2003). A new classification scheme of science fields and subfields designed for scientometric evaluation purposes. Scientometrics, 56 (3): 357-367.

Glänzel, W., y Zhou, P. (2011). Publication activity, citation impact and bi-directional links between publications and patents in biotechnology. Scientometrics, 86 (2): 505-525.

IBM SPSS Statistics Base 19 (2010). C Copyright SPSS Inc. 1989, 2010.

Hinze, S., y Grupp, H. (1996). Mapping of R\&D structures in transdisciplinary areas: new Biotechnology in Food Sciences. Scientometrics, 37 (2): 313-335.

Katz, J. S., y Martin, B. R. (1997). What is research collaboration? Research Policy, 26: 1-18. Klein, J. T. (2008). Evaluation of Interdisciplinary and Transdisciplinary Research. American Journal of Preventive Medicine, 35 (2S): S116-S123.

Langfeldt, L. (2006). The policy challenges of peer review: managing bias, conflict of interests and interdisciplinary assessments. Research Evaluation, 15 (1): 31-41.

Laudel, G. (2006). Conclave in the Tower of Babel: how peers review interdisciplinary research proposals. Research Evaluation, 15 (1): 57-68.

Leydesdorff, L., y Heimeriks, G. (2001). The Self-Organization of the European Information Society: The Case of "Biotechnology". Journal of the American Society for Information Science and Technology, 52 (14): 1262-1274.

McCain, K. (1995). The Structure of Biotechnology R \& D. Scientometrics, 32 (2): 153-175.

Morillo, F., y De Filippo, D. (2009a). Descentralización de la actividad científica. El papel determinante de las regiones centrales: el caso de Madrid. Revista Española de Documentación Científica, 32 (3): 29-50.

Morillo, F.; Aparicio, J., y Bordons, M. (2009b). Informe sobre la Biotecnología en la Comunidad de Madrid (1999-2002 y 2003-2006). Instituto de Estudios Documentales sobre Ciencia y Tecnología (IEDCYT-CCHS), CSIC, Madrid.

Morillo, F.; Aparicio, J., y Bordons, M. (2009c). The Role of Sub-Specialities in the Assessment of Research Teams. En: Larsen, B. y Leta, J.(eds.). Proceedings of ISSI 2009. 12th International Conference of the International Society for Scientometrics and Informetrics. Río de Janeiro, Brasil. 
Morillo, F.; Bordons, M., y Gómez, I. (2003.) Interdisciplinarity in science: a tentative typology of disciplines in research areas. Journal of the American Society for Information Science and Technology, 54 (13): 1237-1249.

Noma, E. (1986). Subject classification and influence weights for 3,000 journals. Report. Computer Horizons, Inc./CHI Research [datos actualizados a 2007 por The Patent Board, nombre actual de CHI Inc.].

Pérez, C. (2005). Técnicas Estadísticas con SPSS (c) 12. Aplicaciones al análisis de datos. Pearson Educación, S.A., Madrid.

Porter, A. L.; Schoeneck, D. J.; Roessner, D., y Garner, J. (2010). Practical research proposal and publication profiling. Research Evaluation, 19 (1): 29-44.

Rey-Rocha J.; Garzon-Garcia, B., y Martin-Sempere, M. J. (2006). Scientists' performance and consolidation of research teams in Biology and Biomedicine at the Spanish Council for Scientific Research. Scientometrics, 69 (2): 183-212.

Rip, A., y Courtial, J. P. (1984). Co-word maps of Biotechnology: an example of cognitive Scientometrics. Scientometrics, 6 (6): 381-400.

Seglen, P. O., y Aksnes, D. W. (2000). Scientific productivity and group size: A bibliometric analysis of Norwegian microbiological research. Scientometrics, 49 (1), 125-143.

van Raan, A. F. J. (2006). Statistical properties of bibliometric indicators: research group indicator distributions and correlations. Journal of the American Society for Information Science and Technology, 57 (3): 408-430.

Vinkler, P. (2000). Evaluation of the publication activity of research teams by means of scientometric indicators. Current Science, 79 (5): 602-612. 